Check for updates

Cite this: RSC Adv., 2017, 7, 43181

2017

Accepted 31st August 2017

DOI: $10.1039 / \mathrm{c} 7 \mathrm{ra06669d}$

rsc.li/rsc-advances

\section{Formation and mechanism of a super-hydrophobic surface with wear and salt spray resistance $\uparrow$}

\author{
Zhi Wang, (D)* Huibin Zhu, Jing He, Yaqing Liu and Guizhe Zhao* \\ A super-hydrophobic surface with excellent wear and salt spray resistance was prepared based on \\ bisphenol A-aniline benzoxazine (BA-a), mesoporous material (SBA-15) and imidazole. The mechanism \\ for these resistances was explored. Density gradient structures, organic-inorganic interpenetrating \\ networks and self-similar structures formed in BA-a/SBA-15/imidazole. The tin sheeting surface coated \\ with BA-a/SBA-15/imidazole hardly changed after 10 days of salt spraying, but its contact angle \\ decreased to lower than $90^{\circ}$. After soaking and heat treatment, the contact angle recovered to $168^{\circ} \pm 2^{\circ}$.
}

\section{Introduction}

Researchers have endeavored to protect against metal corrosion which affects the safety of human life and causes great loss of property. Compared with metals in an air environment, those in the sea are almost bound to undergo corrosion because of numerous free chloride ions in the marine atmospheric environment. The methods for metal corrosion protection mainly include electrochemical protection, coating with anti-corrosion protection materials, etc., wherein introducing a protective coating is an economical and effective method for universal applications. ${ }^{1-3}$ Inspired by the nature of cicada wings, leech legs, rice leaf, lotus, other plants and animals ${ }^{4-7}$ scientists have prepared super-hydrophobic coating materials with similar functions ${ }^{8}$ such as self-cleaning, fouling resistance and corrosion resistance..$^{9-13}$ Highly hydrophobic surfaces ${ }^{14}$ effectively allow drag reduction during navigation and prevent the corrosion of the metal surface. ${ }^{15}$

Although super-hydrophobic coating materials are promising for metal protection, special equipment is required due to the complexity of preparation. ${ }^{16-19}$ Meanwhile, the use of these materials is limited, because they can be easily destroyed owing to micro-nano structures. ${ }^{20}$ Recently, an increasing number of researchers have focused on making robust superhydrophobic surfaces. For example, Huovinen, ${ }^{21}$ Verho ${ }^{22}$ and Paven ${ }^{23}$ have been improved surface mechanical properties by building a multi-level structure. Furthermore, forming chemical connection between low surface energy particles and the modifier is considered as an effective way to improve the mechanical properties of surface. Xue ${ }^{24}$ have been reviewed the self-healing and easily repairable superhydrophobic surface

Research Center for Engineering Technology of Polymeric Composites of Shanxi Province, School of Materials Science and Engineering, North University of China, Taiyuan, 030051, P. R. China. E-mail: shikouri@163.com

$\dagger$ Electronic supplementary information (ESI) available. See DOI: 10.1039/c7ra06669d which can have a prolonged lifetime under the real applications. Dyett ${ }^{25}$ also reviewed the effect of wet chemistry method on mechanical properties of superhydrophobic surface and discussed interplay between chemistry and physical structure which have positive effect on improving the mechanical property of superhydrophobic surface. As seen, different approaches have been reported for fabricating superhydrophobic polymer surfaces with excellent wear resistance. ${ }^{25}$ However, the poor mechanical stability of these superhydrophobic surfaces is still a problem for their practical applications. Therefore, it is of great significance to prepare super-hydrophobic materials with high wear and salt spray resistances by a simple process.

Herein, bisphenol A-aniline benzoxazine (BA-a) with a low surface energy, mesoporous material (SBA-15) and imidazole were used to construct a super-hydrophobic surface through spray coating, giving special density gradient structure, organic-inorganic interpenetrating networks and self-similar structures. The surface showed outstanding wear and salt spray resistances. Additionally, the super-hydrophobic properties of surface after salt spray for 10 days could be recovered by a simple treatment.

\section{Materials and experimental}

BA-a (mp: $113{ }^{\circ} \mathrm{C}$ ) was synthesized and purified according to procedures previously described. ${ }^{26}$ SBA-15 was supplied by Nanjing Xianfeng Nanomaterials Technology Co., Ltd. (China) and used after calcination in a muffle furnace at $400{ }^{\circ} \mathrm{C}$ for $2 \mathrm{~h}$. Imidazole was obtained from Shanghai QianFeng Chemical Co., Ltd. (China). Tin plates were supplied by Hua Electronic Instrument Co., Ltd.

Imidazole (weight ratio: 5\%) was blended with SBA-15 in water and stirred for $10 \mathrm{~min}$. Afterwards, the whole mixture was put into an oven at $70{ }^{\circ} \mathrm{C}$ until the sample was dried. The blend of SBA-15/imidazole (weight ratio: 25\%) and BA-a was dissolved in acetone and sprayed to a glass slide for wear resistance test. 
BA-a/SBA-15 with the same composition was made for comparison. For salt spray test, the mixture was sprayed to tin plates, and pure benzoxazine was also used for comparison. All coated samples were put into an oven after being cured at $120{ }^{\circ} \mathrm{C}$ for $2 \mathrm{~h}$ and $220^{\circ} \mathrm{C}$ for $2 \mathrm{~h}$.

For abrasion test, the prepared surface was loaded with $200 \mathrm{~g}$ force, facing 100 grid SiC sandpaper as an abrading surface, and moved forward in one direction at a rate of $5 \mathrm{~cm} \mathrm{~s}^{-1}$. The gravity of weight was calculated as $3.2 \mathrm{kPa}$ (including weight of the sample). Salt spray tests were done by using a salt spray cabinet (YWX/Q-150, Shanghai Pindun Experimental Equipment Co., Ltd.) according to Chinese National Standard GB/T 1771-91. The surface structures before and after abrasion were studied by scanning electron microscopy (SEM, JSM-6510). The surface morphology was observed and surface roughness was measured by a Brüker Contour GT-X optical profilometer. Water contact angles (WCAs) were measured with a goniometer (KRÜSS DSA
20 , Germany). Droplets of distilled water with a volume of 2-5 $\mu \mathrm{L}$ were placed gently onto the surface at room temperature and pressure. WCAs were measured five times at different locations.

\section{Results and discussion}

To obtain a surface with excellent mechanical performance, imidazole was added into BA-a/SBA-15. Different from common methods, imidazole blended with SBA-15 first, and then the blend was added into benzoxazine. The mechanical properties of cured BA-a/SBA-15/imidazole were evidently improved compared with those of BA-a/SBA-15 (Fig. 1A). BA-a/SBA-15/ imidazole remained super-hydrophobic until sliding more than $240 \mathrm{~cm}$, whereas BA-a/SBA-15 did so within only nearly $100 \mathrm{~cm}$. Before the superhydrophobic property failed, the corresponding SEM images of surface had been showed also. As seen, after wearing, the surface had become smooth, however,
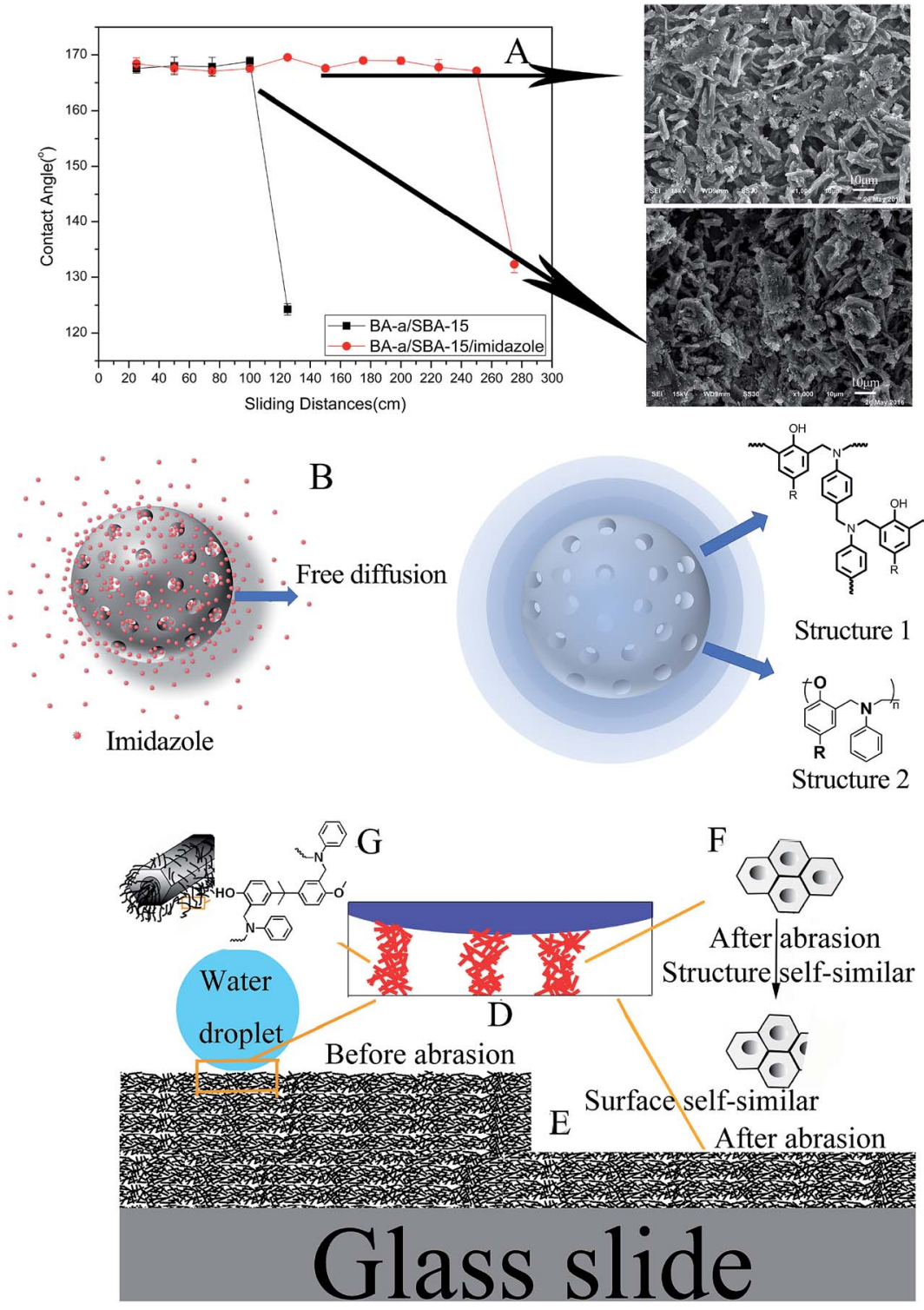

Fig. 1 The WCAs of tin plates coated with BA-a/SBA-15 and BA-a/SBA-15/imidazole (A) and corresponding mechanism (B-G). 
the micro-nano structure still can be observed. This phenomenon may be caused by the self-similar structures as showed below.

The different wear resistance properties of two different system may be attributed to the following two reasons. First, regarding the interface between BA-a and SBA-15, imidazole was free to diffuse because of concentration gradient after SBA-15 was loaded with imidazole and then blended with BAa (Fig. 1B). During curing, imidazole catalyzed benzoxazine to form structure 2 (phenoxy-type structure), and part of benzoxazine which had not been catalyzed formed structure 1 (phenolic-type structure). ${ }^{27}$ Therefore, the whole surface of SBA15 formed a density gradient structure (Fig. 1C, structure 1 and structure 2 have different crosslink densities). Secondly, as to the whole structure, the Cassie's state ${ }^{28}$ was present when water contacted with the surface (Fig. 1D).

In addition, the surface and mesoporous $\mathrm{SiO}_{2}$ had selfsimilar structures before and after abrasion (Fig. 1E and F). Furthermore, benzoxazine bound both the inside and surface of mesoporous $\mathrm{SiO}_{2}$, forming an interpenetrating network (Fig. 1F). The density gradient interfaces, organic-inorganic interpenetrating network and self-similar structures were all responsible for the satisfactory mechanical properties.

Fig. 2A shows the samples before salt spray test. Each piece of tin plate was divided into three layers. The upper layer was sprayed with BA-a/SBA-15/imidazole blends, the middle was sprayed with pure benzoxazine and the bottom was blank. The middle layer failed to completely cover the surface of tin plate because of low surface energy. After SBA-15/imidazole was added into BA-a, it completely infiltrated the tin plate and formed a completely covered protective layer. Thus, SBA-15/ imidazole managed to modify the surface energy of BA-a, enabling the use of benzoxazine as coating. Fig. 2B shows the surface resistance to salt spray after different test times. For middle and bottom layers, the corrosion area gradually expanded with increasing time. For the upper layer, the surface did not change after 10 days of salt spray test, indicating that the blend had high corrosion resistance. However, the surface hydrophobic characteristics changed (Fig. 2C). WCAs markedly reduced from $172^{\circ}$ (super-hydrophobic), $102^{\circ}$ (hydrophobic) to $<90^{\circ}$ (hydrophilic). After salt spray for different times, the samples were placed into deionized water for two days and thereafter put into an oven at $200{ }^{\circ} \mathrm{C}$ for $1 \mathrm{~h}$. As a result, WCAs of the surfaces recovered to more than $165^{\circ}$ (Fig. 2D).

To explore the causes of hydrophobicity changes, different stages of surfaces were examined by SEM (Fig. 3) and white light interference (ESI Table $1 \dagger$ ). As exhibited in Fig. 3A, the uniform structure of rod-like SBA-15 stacks on each other, a micro-nano porous structure uniformly forms between the rod and the stack, and the surface contact angle is $170^{\circ}$. After salt spray for 10 days (Fig. 3B), the rod on surface becomes shorter and unordered, still having the micro-nano porous structure. The surface contact angle becomes $80^{\circ}$. After soaking and heat treatment, the surface morphologies (Fig. 3C and D) are similar to that without soaking (Fig. 3B), and the surface contact angles are $97^{\circ}$ and $165^{\circ}$, respectively. Fig. $3 \mathrm{~A}^{\prime}-\mathrm{D}^{\prime}$ show that the local structures in different states are similar, as also evidenced by the surface roughnesses (Fig. $3 \mathrm{~A}^{\prime \prime}-\mathrm{D}^{\prime \prime}$, and ESI Table $1 \dagger$ ). The change of surface hydrophobicity was not related with the surface morphology, probably due to salt spray test. During this test, sodium chloride that bound the surface and pores was left on the surface after evaporation of water.

To verify the above assumptions, the elements of surfaces at different stages were detected (ESI Table $2 \dagger$ ). After salt spray test, the material surface had considerable chlorine and sodium elements (over 14\%). These elements significantly decreased after soaking $(\mathrm{Na}, 0.583 ; \mathrm{Cl}, 0.146)$ and further did so after heat treatment (Na, 0.083; Cl, 0.065). Similar to previous analysis, the content of $\mathrm{NaCl}$ on the surface of blend system determined the surface hydrophobicity. Especially, the differences between C and $\mathrm{D}$ may be ascribed to different water evaporation rates, so different contents of $\mathrm{NaCl}$ were left.

In order to illustrate the mechanism of corrosion inhibition property and restorability of surface, illustration image was showed as Fig. 4. After spraying and curing, the glass surface was coated with designed materials. The designed materials can inhibit other materials contact with glass surface and can protect the surface from corrosion. After salt spray, the $\mathrm{NaCl}$ can
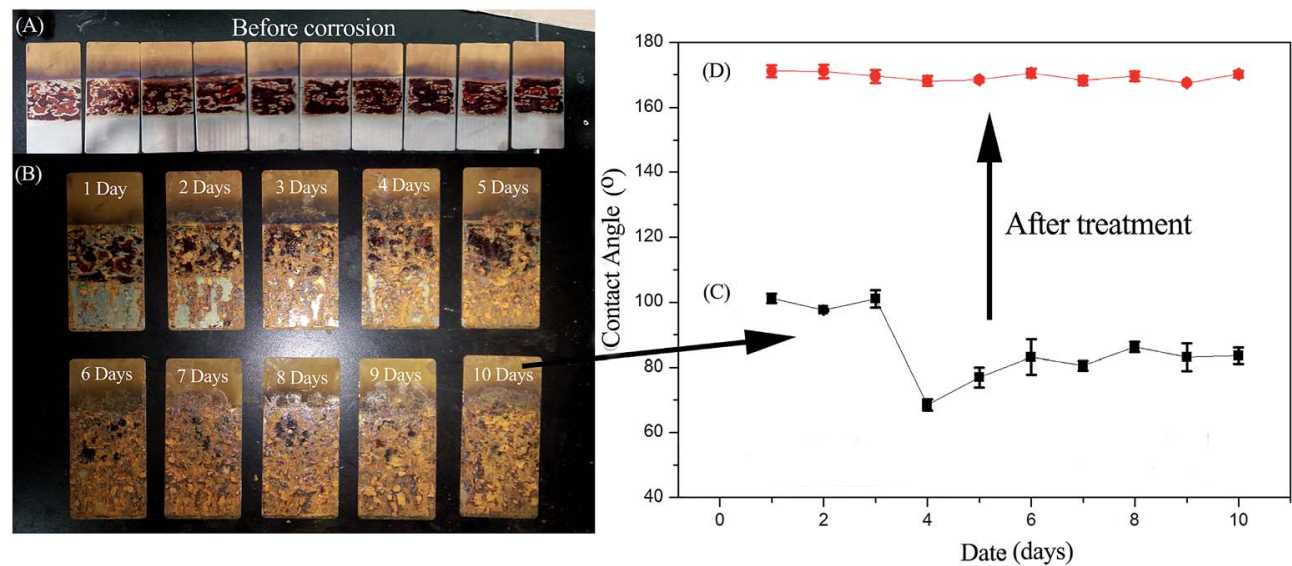

Fig. 2 The surfaces of tin plates before (A) and after (B) salt spray test, the water contact angles of tin plates after different days' salt spray test (C) and after placing into deionized water and heated at $200^{\circ} \mathrm{C}$ (D). 

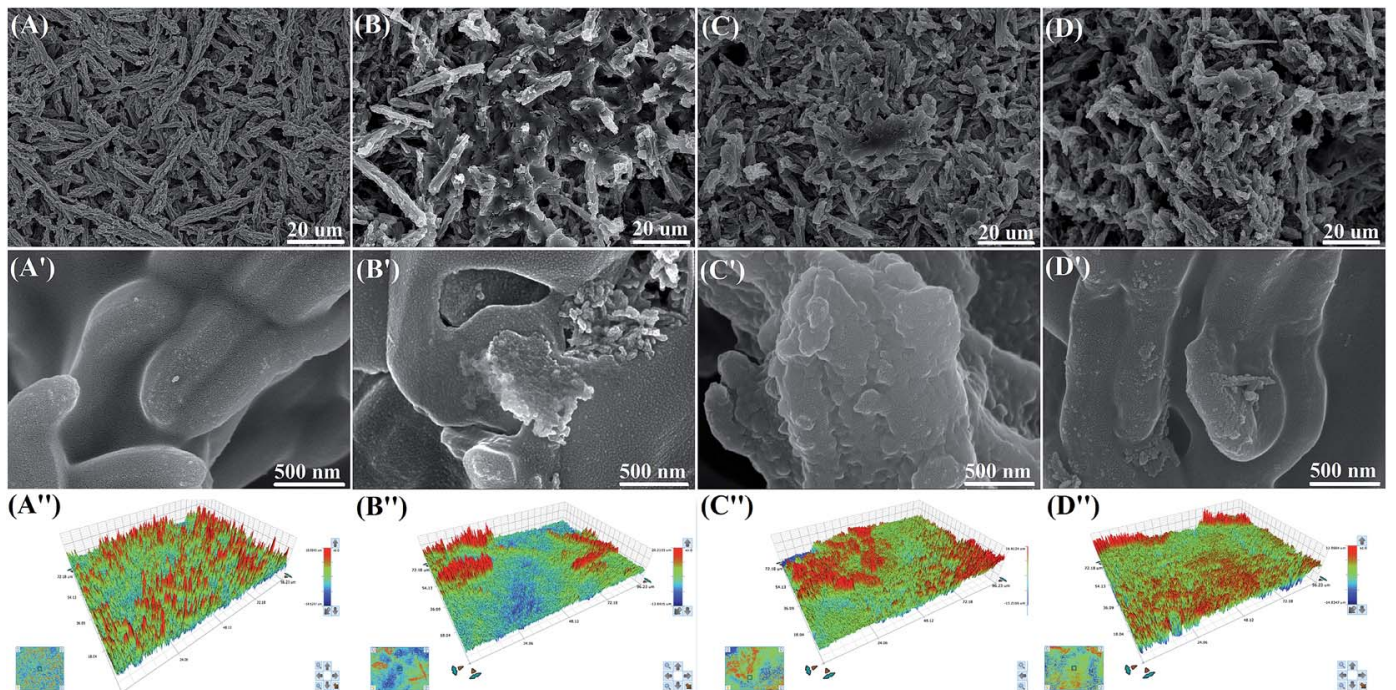

(B")

(C")

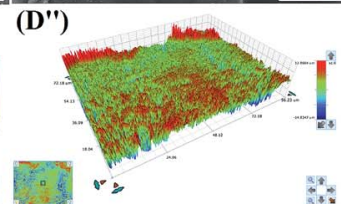

Fig. 3 SEM images and 3D view of micro scale structures ( $A, A^{\prime}$ and $\left.A^{\prime \prime}\right)$, surface before salt spray test; ( $B, B^{\prime}$ and $\left.B^{\prime \prime}\right)$, surface after 10 days' salt spray test; $\left(C, C^{\prime}\right.$ and $\left.C^{\prime \prime}\right)$, surface after placing into deionized water; $\left(D, D^{\prime}\right.$ and $\left.D^{\prime \prime}\right)$, surface after heated at $\left.200{ }^{\circ} \mathrm{C}\right)$.

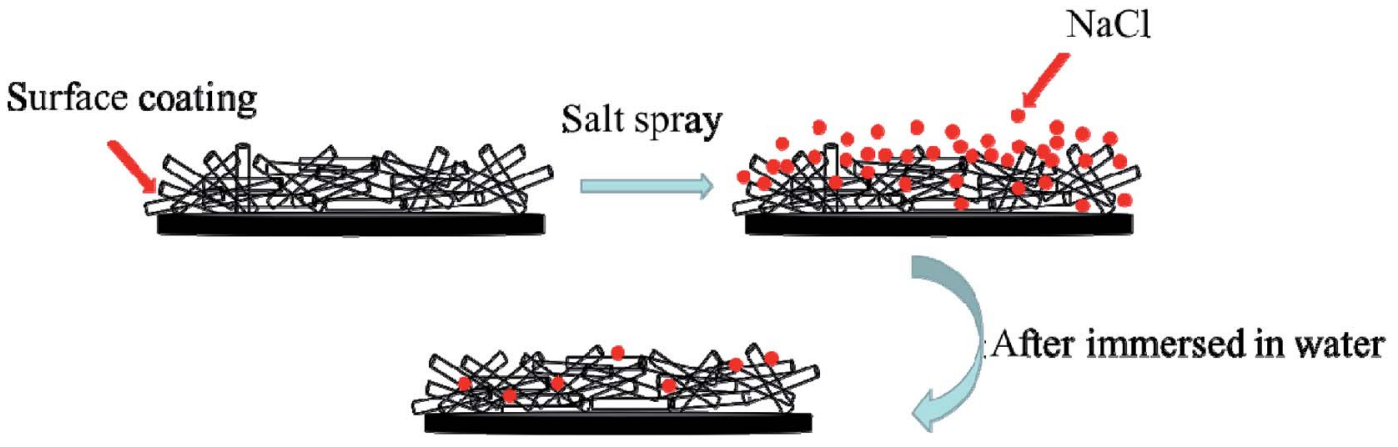

Fig. 4 Illustration image of corrosion inhibition property and restorability of surface.

attach in the micro-nano surface, the property of whole surface changed from superhydrophobic to hydrophilic. Because of the excellent wear resistance properties of coated surface, the morphology of micro-nano surface had no change after immersed in water or washed by water as shown in Fig. 4 . Meanwhile, because of the $\mathrm{NaCl}$ was washed by water, the coated surface changed from hydrophilic to superhydrophobic again.

\section{Conclusions}

Super-hydrophobic surface with excellent wear and salt spray resistances was made based on BA-a/SBA-15/imidazole. The corresponding mechanism was clarified. Density gradient structure, organic-inorganic interpenetrating networks and self-similar structures were responsible for the high wear resistance. For BA-a/SBA-15/imidazole, the surface morphology did not change during salt spray test, and whether sodium chloride existed on surfaces affected the hydrophobicity.

\section{Conflicts of interest}

There are no conflicts to declare.

\section{Acknowledgements}

Financial support was provided by the National Natural Science Foundation of China (Project No. 51503187 and No. 51773185), Project 201601D021058 supported by Natural Science Foundation of Shanxi.

\section{References}

1 D.-M. Zang, D.-K. Cao and L.-M. Zheng, Inorg. Chem. Commun., 2011, 14, 1920.

2 A. L. Shao, Y. Cheng, Y. Zhou, et al., Surf. Coat. Technol., 2013, 228, S257.

3 X. Deng, L. Mammen, H.-J. Butt and D. Vollmer, Science, 2012, 335, 67.

4 T. Sun, L. Feng, A. Xuefeng Gao and L. Jiang, Acc. Chem. Res., 2005, 38, 644 . 
5 X. Gao and L. Jiang, Nature, 2004, 432, 36.

6 S. Pan, A. K. Kota, J. M. Mabry and A. Tuteja, J. Am. Chem. Soc., 2013, 135, 578.

7 X. Zhang, J. Zhao, Q. Zhu, N. Chen, M. Zhang and Q. Pan, ACS Appl. Mater. Interfaces, 2011, 3, 2630.

8 K. Liu and L. Jiang, Nanoscale, 2011, 3, 825.

9 M. Kobayashi, Y. Terayama, H. Yamaguchi, et al., Langmuir, 2012, 28, 7212.

10 I. Milošev, T. Kosec and M. Bele, J. Appl. Electrochem., 2010, 40, 1317.

11 H. Jin, M. Kettunen, A. Laiho, et al., Langmuir, 2011, 27, 1930.

12 G. Kwon, A. K. Kota, Y. Li, A. Sohani, J. M. Mabry and A. Tuteja, Adv. Mater., 2012, 24, 3666.

13 T. Sun, L. Feng, X. Gao and L. Jiang, Acc. Chem. Res., 2005, 38, 644.

14 C. J. Weng, C. H. Chang, I. L. Lin, et al., Surf. Coat. Technol., 2012, 207, 42.

15 V. B. Mišković-Stanković, M. D. Maksimović, Z. KačarevićPopović and J. B. Zotović, Prog. Org. Coat., 1998, 33, 68.

16 S. Feng, Z. Zhong, F. Zhang, Y. Wang and W. Xing, ACS Appl. Mater. Interfaces, 2016, 8, 8773.
17 J. Xi, L. Feng and L. Jiang, Appl. Phys. Lett., 2008, 92, 053102. 18 S. G. Lee, D. S. Ham, D. Y. Lee, H. Bong and K. Cho, Langmuir, 2013, 29, 15051.

19 H. Zhou, H. Wang, H. Niu, J. Fang, Y. Zhao and T. Lin, Adv. Mater. Interfaces, 2015, 2, 1400559.

20 H. Zhou, H. Wang, H. Niu, Y. Zhao, Z. Xu and T. Lin, Adv. Funct. Mater., 2017, 1604261.

21 E. Huovinen, J. Hirvi, M. Suvanto and T. A. Pakkanen, Langmuir, 2012, 28, 14747.

22 T. Verho, C. Bower, P. Andrew, S. Franssila, O. Ikkala and R. H. A. Ras, Adv. Mater., 2011, 23, 673.

23 M. Paven, R. Fuchs, T. Yakabe, et al., Adv. Funct. Mater., 2016, 26, 4914.

24 C.-H. Xue and J.-Z. Ma, J. Mater. Chem. A, 2013, 1, 4146.

25 B. P. Dyett, A. H. Wu and R. N. Lamb, ACS Appl. Mater. Interfaces, 2014, 6, 18380.

26 D. Pei, G. U. Yi and X. Cai, Acta Polym. Sin., 1998, 5, 595.

27 C. Liu, D. Shen, R. M. Sebastián, J. Marquet and R. Schönfeld, Macromolecules, 2011, 44, 4616.

28 S. Wang and L. Jiang, Adv. Mater., 2007, 19, 3423. 